\title{
Mathematical Analysis of Aerodynamic Potential of Ebola Virus Particle
}

\author{
David Blackman Hon.
}

Mathematics Department, Southern Oregon University, USA

Copyright@2018 by authors, all rights reserved. Authors agree that this article remains permanently open access under the terms of the Creative Commons Attribution License 4.0 International License

\begin{abstract}
In aerodynamics, there are two types of surfaces: stiff and flexible. Stiff surfaces may gain energy from differential wind velocity on opposite sides of the particle. Flexible surfaces dissipate such energy through flutter i.e. like the tail of a kite. Kites have two components, the airfoil and the tail. Airfoils provide lift for the kite through the differential air currents. In such a dichotomous world the Ebola virus resembles the tail of the kite as opposed to the airfoil. One would expect the Ebola virus to flutter and fall. Through mathematical analysis of the electron micrograph it is concluded that the virus is not airborne, but it is probably waterborne.
\end{abstract}

Keywords Ebola, Aerodynamics, Mathematical Modeling, Electron Micrograph of Ebola

\section{Introduction}

The electron-micrograph of two Ebola viruses, notice how different the two Ebola viruses appear. Viruses in figure 1 are airborne viruses and as one can see it has many rigid structures which mean no flapping in the wind stretched over airframes, which renders them rigid and aerodynamic. The point here is that aircraft wings are aerodynamic stiffened surfaces which prevent wing aeroelastic flutter.

Imagine a violin string with a note played on it. Now also imagine the string in mid note is seen through high-speed photography. One would expect to see a waveform on the string. There would be nodal points in both high and low extrema. Now imagine cutting at both ends of the string in mid-note. The string would pop off the violin in a tangle. One could imagine the Ebola virus as such a tangle. Examine the two viruses in figure 2 and visualize these two viruses as liberated violin strings. The usual relationships in physics applied, wavelength time's frequency should equal the speed of sound.

\section{VIRAL SHAPES}

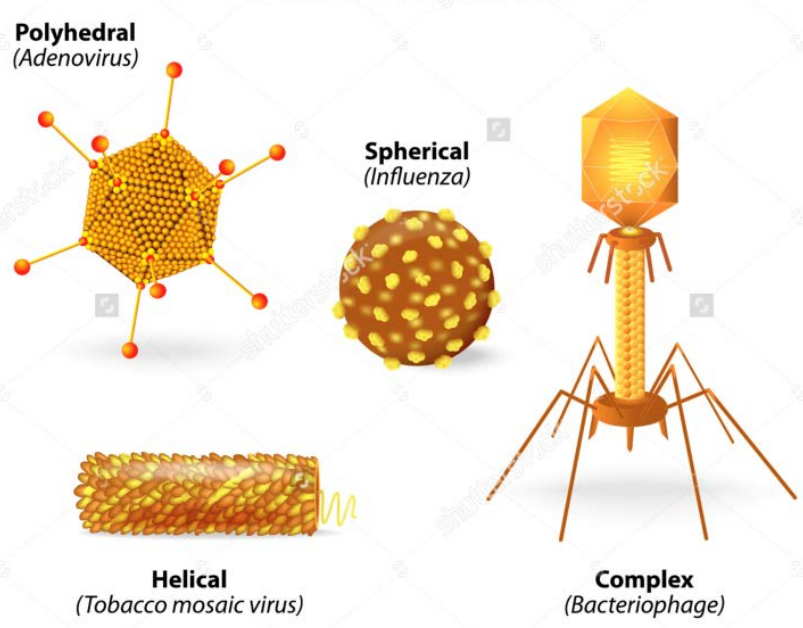

Figure 1. Viruses in are airborne viruses and as Viruses in figure 1 are airborne viruses. [1]

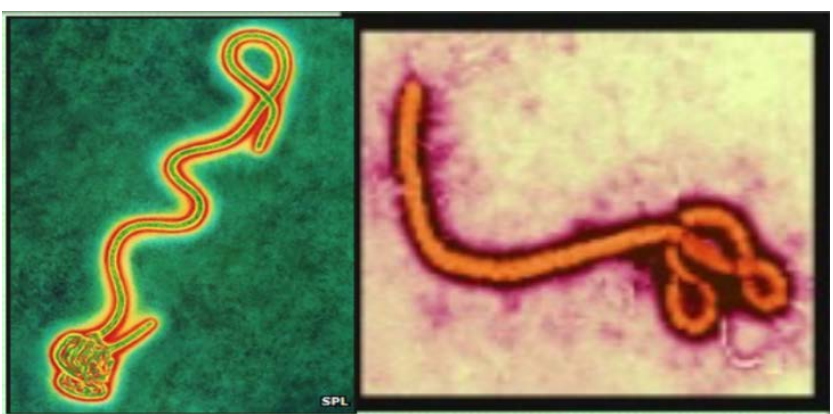

Figure 2. The first Electron micrograph of Ebola Zaire virus [2]

There has been much speculation in the media that Ebola may be an airborne virus. Airborne viruses seem to have stiff surfaces, which is not true of Ebola. To address this question, it is necessary to elucidate what makes something aerodynamic as opposed to hydrodynamic. The Bernoulli equation as shown below has three terms, each representing a mode of importing energy to the system. The three terms in the Bernoulli equation correspond to potential energy that is involved in energy of elevation, 
energy of environmental interactions, and kinetic energy of fluid dynamics. It is this last term which is critical. It is this kinetic energy term which is most effective by fluctuating materials.

In aircraft, fabric wings are doped to stiffen the surface with plasticizer material. Early aircraft used cotton and cellulose nitrate. Modern fabric-covered designs usually use synthetic materials such as Dacron and butyrate. This method is often used in the restoration of older cloth wing, it tightens and stiffens fabric. The Steve Wittman crash is a typical and classic example of what can go wrong. [3] Wittman had designed a racing plane which renders them rigid and aerodynamic. [3] The point here is that aircraft wings are aerodynamic stiffened surfaces which prevent wing aeroelastic flutter the electron-micrograph of two Ebola viruses, notice how different the two Ebola viruses appear. One can see it has many rigid structures which means no flapping in the wind stretched over airframes, which renders them rigid and aerodynamic. With a pair of doped cloth wings, Steve Wittman's plane crashed. [3] The doping failed and the plane crashed, killing both him and his wife. [3] What apparently happened to Wittman's aircraft was a phenomenon known as aerodynamic flutter. Fluttering of the wing surface dissipates the energy of elevation resulting in a crash. The point here is that aircraft wings are aerodynamic stiffened surfaces which prevent wing aeroelastic flutter.

\section{Materials and Methods}

The electron-micrographs are inserted into a file on Painter 12.0 [4] and digitized with the pixel locating function of Corel Painter. [5] Each virus electron-micrograph yields two data sets, the meandering is both the $\mathrm{X}$ and $\mathrm{Y}$ coordinate axis, which are fitted to two parametric polynomials by KaleidaGraph. These polynomials are further analyzed with the Fast Fourier Transform algorithm in KaleidaGraph [M].

Micrographs illustrate the fact that the Ebola virus appears to have a rod-shaped morphology which can twist and overlap itself. The measured length of the virus is 1400 $\mathrm{nm}$ in Length with a diameter of $80 \mathrm{~nm}$ in diameter. In biological systems, most things are made of proteins, and RNA or DNA is plastic. Ebola is a combination of both RNA and DNA. Typically proteins and polynucleotides are rubber-like materials unless there is something present to give them rigidity. Likewise DNA and RNA are also rubber-like materials and unless there is some kind of structural integrity, objects made from these materials tend to be non-rigid. Flu virus above (figure 1) is a perfect example of an object with rigidity owed to spherical symmetry. The Bacteriophage virus on the other hand, has a complex structure with many rigid parts. Bacteriophage Virus BV has a stiff head above a cylindrical body with feet below. The binding site for this virus on the cells that BV attaches is located at the base of the feet. The virus most similar to the Ebola virus in figure 1 is the Tobacco Mosaic virus. The case of the Tobacco Mosaic will be addressed in the results section.

This treatment of the TEM is analogous to a photo of a violin string clipped in mid-note. These plots are evidence of flutter. What the Fast Fourier Transforms (FFT) provides evidence of, is that there are a multitude of vibronic modes. One interpretation of these results would be a collection of longitudinal and lateral vibronic modes with a 17.5 to one virus on the cells that BV attaches is located at the base of the feet. The virus most similar to the Ebola virus in figure 1 is the Tobacco Mosaic virus. The case of the Tobacco Mosaic will be addressed in the results section.

The micrograph is of the Ebola virus. This micrograph illustrates the fact that the Ebola virus has a rod-shaped morphology which can twist and overlap itself. The question here is how to measure the degree of flexibility in this virus. As one can clearly see from the micrograph, the virus is quite flexible and would tend to dissipate energy in unproductive flapping motions. The aerodynamic properties of such a structure one should expect to be minimal. It would be like a kite with all tail and no airfoil. The problem becomes one of how to quantify this property. By definition, a perfect airfoil would have zero flexibility. This is the proposed methodology for quantifying the flexibility factor.

One problem obviously is that it is impossible to get depth perception on an electron micrograph.

Since the Ebola virus is a rod-shaped particle with twists and turns the electron micrograph will be imposed upon a two-dimensional Cartesian axis. A central path is drawn over the image using the Pixel tracking function in Corel Painter 12.0. The ordered pair of $\{\mathrm{X}, \mathrm{Y}\}$ coordinates are fitted to an ordered pair of polynomials $\{P(x), Q(y)\}$. These will be parametric relationships between increments of path length. The data is digitalized by placing dots along the image of the Ebola virus. KaleidaGraph by Synergy is the software used to analyze the data fitting to Ninth degree polynomials. These Polynomials are fit from the data with $\mathrm{R}>$ 0.99. Digitalized plots of the optical images are in figure 3 . The data has the minimum value point subtracted from all data points, and this is equivalent to moving the viral TEM towards the upper corner. Fast Fourier Transforms are done on these polynomials as a final step. This is done by KaleidaGraph. The Fast Fourier Transform (FFT) which was documented by Neil Singer, for Convolve, Inc. (Copyright 1990 by Convolve, Inc.) [6] 

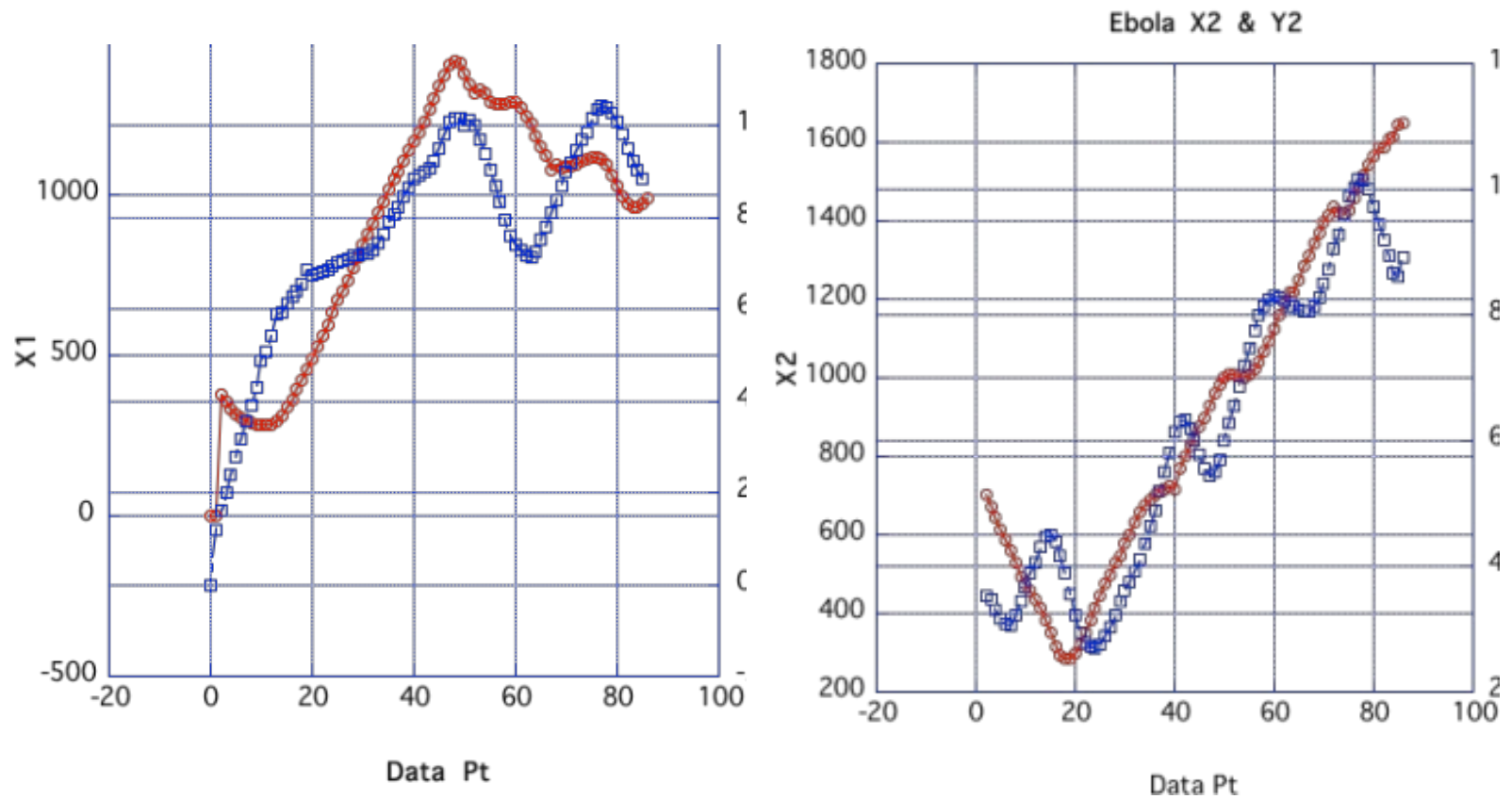

Figure 3. Plots of the parametric Polynomials for two Ebola viruses, Polynomial Parametric Decomposition of the two Ebola virus electron micrograph.

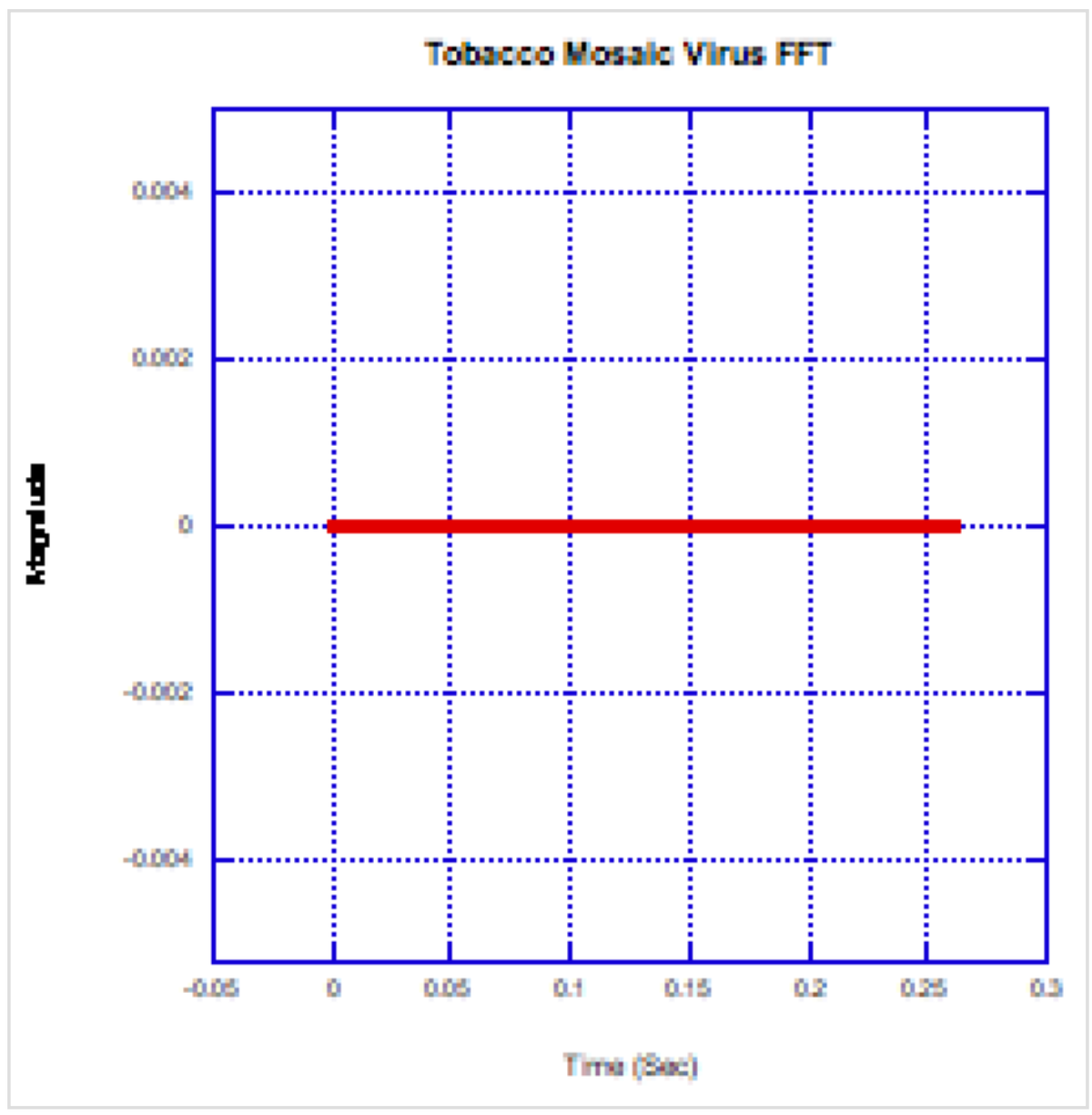

Figure 4. Fast Fourier Transforms for Tobacco Mosaic and the two Ebola virus electron micrographs 


\section{Results}

The process de-convolutes the meanderings on the $\mathrm{X}$-axis from those on the $\mathrm{Y}$-axis. 9th degree polynomials are employed to derive good fits. $\mathrm{R}>0.99$. The polynomial plots above are the polynomials set for these two Ebola TEM analyzed. The plots below are Figure 5 Fast Fourier Transforms of the polynomials derived from the TEM of the two Ebola viruses.

One interpretation of these results would be a collection of longitudinal and lateral vibronic modes with a 17.5 to one ratio between the longitudinal and lateral prime frequencies. This would reflect the diameter to length ratio of Ebola being 17.5 to one.

The length of the virus is $1400 \mathrm{~nm}$ and the diameter is 80nm. In all of these FFT there are high frequency virbonics and low frequency vibronics which explain the U-shaped FFT curves. Figure 3. The special case of the Tobacco Mosaic Virus, illustrates the difference between the stiff airborne virus Tobacco Mosaic Virus and the plastic Ebola Virus. The principle difference between TMV and EV is the ability of EB to dissipate energy in flutter modes. The parametric polynomial for TMV is of course a straight line, which results in no flutter in the Fast Fourier Transform.

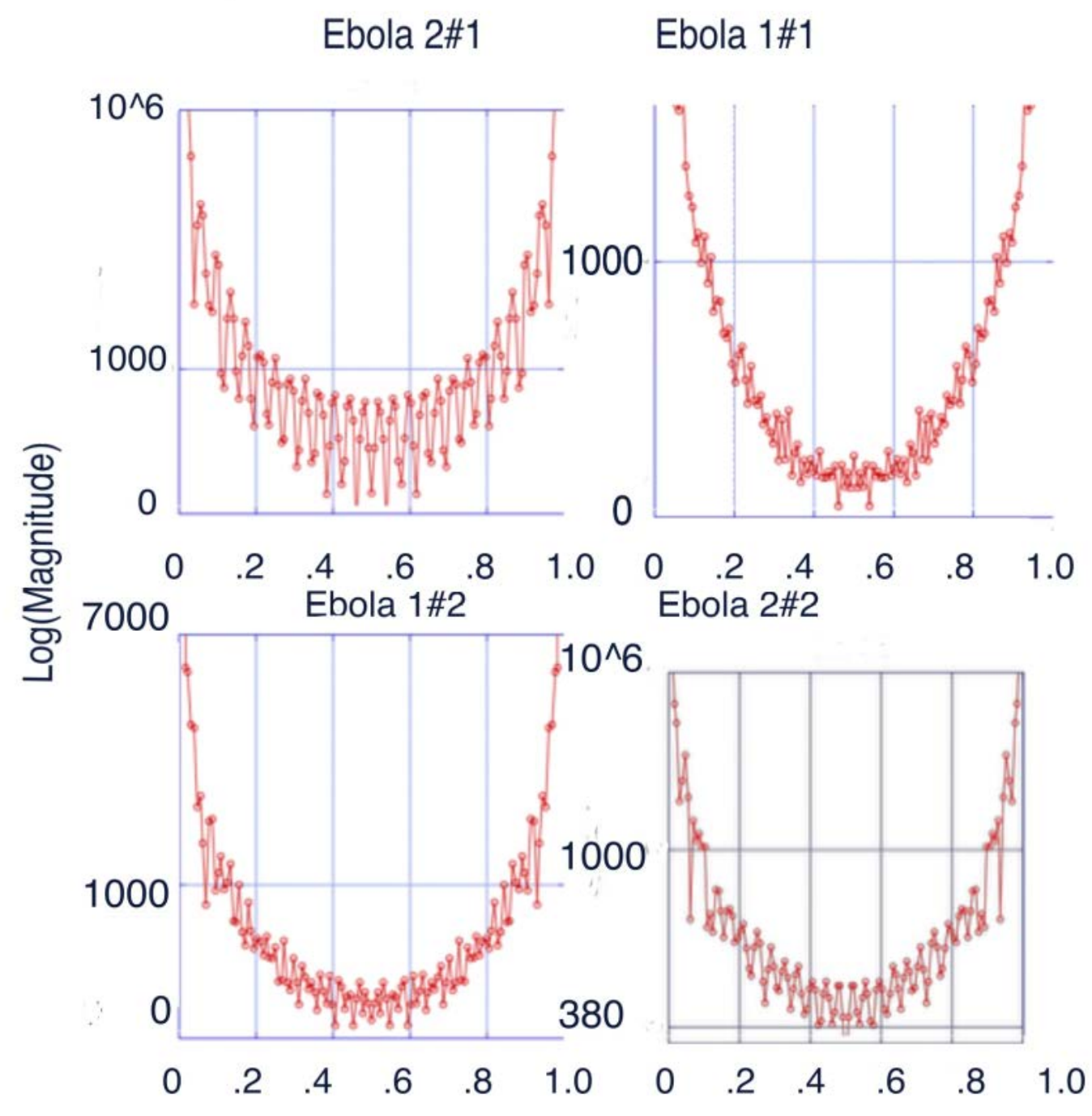

Figure 5. Fast Fourier Transforms of the polynomials derived from the TEM of the two Ebola viruses 


\section{The Bernoulli Equation}

$$
\frac{P}{\rho}+\frac{v^{2}}{2}+g h=C
$$

The three terms in the Bernoulli equation are the buoyancy term i.e. ratio of pressure to density; air velocity term; and gravity potential energy term. Since the density of protein and nucleic acid are comparable to water but much greater than air. Thus, the buoyancy of the virus is potentially positive in water but definitely negative in air. The density ratio compared to air is 2 orders of magnitude greater than air. This negative buoyancy in air and gravity acts to pull the particle downward. The only term in the equation which can provide lift is the differential air velocity term. This is the term effected by flutter or flapping.

Kinetic energy gain is caused by air velocity differential on opposing sides. Flutter dissipates this energy.

If one does this procedure on a straight line Tobacco Mosaic Virus one gets a string of zeros for the magnitude of the frequency components of the FFT. There are no flutter frequencies which will dissipate potential energy of elevation in FFT of the Tobacco mosaic virus.

\section{Conclusions}

One obvious conclusion is of the difference between tobacco mosaic virus versus Ebola virus. It is precisely the intuitive expected result. Tobacco mosaic is a stiff virus with no vibronic modes apparent in the FFT. Ebola on the other hand has a plethora of vibronic states (in its FFT's. Each vibronic mode is a depository for the viral particles to dump energy. The shape of the FFT curves is as one would expect. There are collections of high-frequency vibronics, possibly associated with the transverse waves across the virus and some low-frequency attributable to the longitudinal waves running along the length of the virus. The ratio of length versus width for Ebola is about 17.5 to 1 . It is not much of a stretched imagination to visualize the Ebola virus being much like the tail of a kite. This means that the FFT is caused by the present set of wiggles in the electron micrograph. Tobacco mosaic virus is known to be an airborne virus and it exhibits no flutters. This conclusion doesn't apply to transport in water because the buoyancy term. In an aqueous medium, the pressure versus density terms is likely either a positive buoyancy or if negative is very small in magnitude. That's the likely route of transmission of Ebola is liquid i.e. water borne.

So the Ebola virus is a string. The evidence for that is the plethora of detectable vibronic modes. One could ask the question why not extend the analysis to detect this elastic nature of the virus and the density of virus and the whole plethora of other things buried in the data. The answer is one simple, much information is lost to the third dimension loss because the projection of the electron micrograph qualities questions. As a question of economics, when one sees healthcare workers in Western Africa wearing minimal protective clothing versus what one saw in Dallas Texas circa 2014. Healthcare workers in Africa were facing the real thing the real virus. Healthcare workers in Dallas Texas were confronted with the hysteric the monster virus, airborne and destine to depopulating the planet.

Clearly the evidence indicates space suit response was rather melodramatic. Yes, Ebola is a serious disease. Is there any chance of becoming airborne, not likely! The economic, simply the cost of an airtight spacesuit verse the cost of a more modest protective garb. Adequate water treatment might prove economically more effective than spacesuits. Mathematics of Ebola structural clearly indicates this virus is not likely going to become airborne. Similar viruses like Magdeburg which has a similar electron micrograph are also not likely to become airborne.

There are other considerations, aspects which are not controllable in a strictly mathematical treatment. Electron micrographs were probably done on a virus particle extracted from aqueous solution. The vibronic modes evident in the Fast Fourier Transform are affected by this experimental consideration. Ultimately, what one sees in the parametric polynomials are oscillations directly connected to the interaction between suspending medium (read aqueous solution). It is highly unlikely that the virus fell out of the sky. Far more likely scenario is that the virus fell out of suspension. That fact alone makes interpretation of the Fast Fourier Transform results not indicative of what would happen with virus in air. However, if the virus flutters of aqueous solution it is safe to assume that the virus is flexible or in other words plastic. In air the expected result would be higher frequency flutter.

From this analysis, it is best to restrict conclusions to the question of flexibility of the virus. The usual questions which this type of analysis would normally address such as virus density and composition are not answered by this analysis. The only question that this analysis addresses is the aerodynamic properties of the virus. The crucial an economically important consideration is the issue can Ebola fly? To this question, it is safe to say that Ebola is not airborne capable and is more like the tail of the kite more than the airfoil of said kite.

\section{Acknowledgements}

Thanks to Daniel Alrick for editorial support and Alex Berry for mathematical editing. 


\section{Appendix}

\begin{tabular}{|c|c|c|c|c|}
\hline \multicolumn{5}{|c|}{ Parametric Polynomials Ninth Degree } \\
\hline $\mathrm{P}(\mathrm{X}$ or $\mathrm{Y})$ & $\mathrm{X} 1$ & $\mathrm{Y} 1$ & $\mathrm{X} 2$ & $\mathrm{Y} 2$ \\
\hline M0 & 344.05 & -10.029 & 714.89 & 392.55 \\
\hline M1 & 32.308 & 95.172 & -12.721 & -61.046 \\
\hline M2 & -15.228 & -16.499 & -3.3071 & 16.236 \\
\hline M3 & 1.9991 & 2.1706 & 0.16657 & -1.6472 \\
\hline M4 & -0.12067 & -0.14891 & 0.0041825 & 0.082921 \\
\hline M5 & 0.0041092 & 0.0055832 & -0.0004161 & -0.0023266 \\
\hline M6 & $-8.296 \mathrm{E}-05$ & -0.0001196 & 1.0821E-05 & 3.8304E-05 \\
\hline M7 & 9.7919E-07 & $1.4608 \mathrm{E}-06$ & $-1.3488 \mathrm{E}-07$ & $-3.6824 \mathrm{E}-07$ \\
\hline M8 & $-6.2267 E-09$ & $-9.4608 E-09$ & 8.3203E-10 & 1.9145E-09 \\
\hline M9 & 1.6455E-11 & $2.5204 \mathrm{E}-11$ & $-2.0428 E-12$ & $-4.1601 \mathrm{E}-12$ \\
\hline $\mathbf{R}$ & 0.9982 & 0.99642 & 0.99379 & 0.98019 \\
\hline
\end{tabular}

\section{REFERENCES}

[1] https://www.dreamstime.com/stock-illustration-shapes-viru ses-viral-vector-illustration-showing-there are-many-different-sizes-image43457161

[2] Dr. F.A. Murphy, CDC. 160,000 x magnification and another TEM. There is little doubt that these two viruses are not congruent in the geometric sense. The electron-micrograph of two Ebola viruses.

[3] Chicago Tribune May 1, 1995. The crash was caused by delamination of the wings, evident by the scattered debris over miles of mountainous terrain in Alabama.

[4] Painter 2017: 9 Wells Avenue, Suite 300 Yonkers, NY 10701

[5] Kaleida Graph: by Synergy Software, Version 4.5.2 April 24,2014 Copyright 1986-2014

[6] The Fast Fourier Transform (FFT): which was documented by Neil Singer, for Convolve, Inc. (Copyright 1990 by Convolve, Inc.) 